\title{
Dielectric Strings: A Low Clutter Method for Bistatic RCS Measurements
}

\author{
Peter J. Collins \\ Department of Electrical and Computer Engineering \\ Air Force Institute of Technology \\ Wright-Patterson AFB, Ohio 45433 \\ Email: peter.collins@afit.edu
}

\begin{abstract}
Bistatic RCS measurements have seen a recent resurgence as interest in passive coherent location (PCL) systems and other survivable detection schemes has increased. Low RCS bistatic measurements in particular provide a unique challenge to the RCS metrologist as noise, clutter, and interfering signals become significant relative to the desired signal. Traditional methods for supporting the bistatic antenna/receiver often provide a significant source of moving clutter that interacts strongly with the target. This paper presents a new low clutter method based on recent advances in string-based target support systems. A simple experiment is described and the resulting fixed angle bistatic measurement results are qualitatively analyzed, clearly demonstrating the benefits of the proposed methodology.
\end{abstract}

\section{INTRODUCTION}

Bistatic radars and consequently bistatic Radar Cross Section (RCS) measurements have existed from the advent of radar nearly 80 years ago. During that period, interest in bistatic RCS metrology has ebbed and flowed in an approximate fifteen year cycle depending on the perceived advantages for specific applications over the ubiquitous monostatic techniques [1]. Recently interest has turned to bistatic radar again as a possible means to provide a survivable counter-stealth capability [2]. Low RCS measurements provide a unique challenge to the RCS metrologist as many of the contaminates such as noise, clutter, and interfering signals, safely ignored for relatively high RCS targets, become significant sources of measurement uncertainty. The issue is compounded for bistatic measurements as the clutter becomes a function of the bistatic angle and often the receiver structure itself.

This paper presents a new mitigation strategy aimed at solving this problem by reducing the clutter associated with the bistatic antenna support structure. The concept leverages the lessons learned from recent string-based target support designs [3]. Key among these is the fact the stability of the inverted Stewart platform far exceeds the traditional three and four string designs, providing a very stable, low interaction support system for RCS measurements [4]. These same properties are exactly what is needed to reduce clutter introduced by the antenna support structure in a bistatic measurement.

The following paragraphs develop this idea in the context of an indoor bistatic measurement. After briefly discussing the concepts related to bistatic RCS measurements, the paper describes the method whereby a string-based inverted Stewart platform is used to suspend the bistatic receiver/antenna assembly of a bistatic measurement radar. This approach is compared to the traditional method of supporting the assembly with an absorber coated tower. Next an experiment is described with the intent of demonstrating the benefits of the proposed design in reducing the measurement uncertainty. The measurement results for a 6" square metal plate are presented showing a significant improvement in measurement quality.

\section{Bistatic RCS And Bistatic Measurements}

Bistatic RCS is defined by the IEEE Std 686-2008 as one of the cases of the more general radar cross section (RCS) definition whereby energy is "reflected or scattered in any direction other than the incident direction or opposite of the incident direction." According to this standard, RCS is "a measure of the reflective strength of a radar target; usually represented by the symbol $\sigma$ and measured in square meters." [5]. The reflective strength is not only a function of physical size, but of shape, frequency, polarization, aspect angle, and target material properties.

Figure 1 depicts a typical bistatic measurement configuration. Notice for any target position there are associated lines of

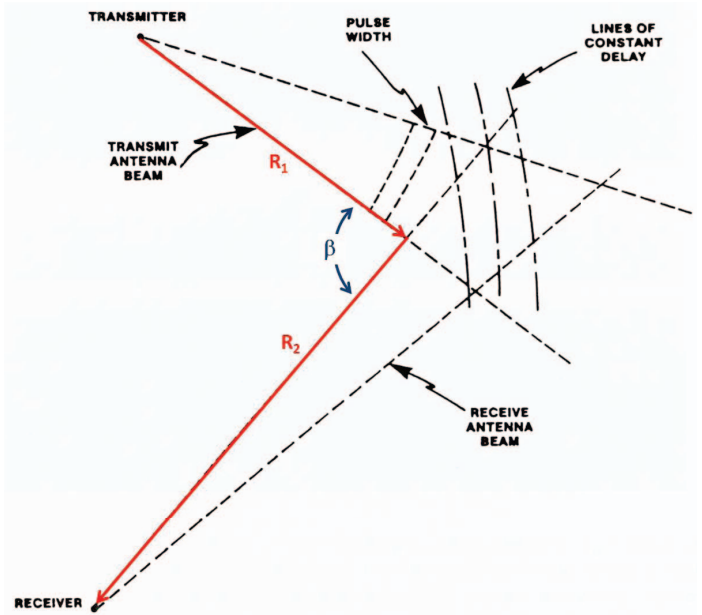

Fig. 1. Bistatic Measurement Geometry with Bistatic Angle $\beta$ [7]

constant phase delay where scattering from the measurement environment can be introduced through antenna side lobes or target bistatic illumination to provide a contamination 
source. Equation (1) gives the received power for the case of distributed clutter characterized by an RCS per unit area $\sigma^{c}$ as,

$$
P_{r}=\frac{P_{t} \lambda^{2}}{(4 \pi)^{3}} \int_{A} \frac{G_{t} G_{r} \sigma^{c}}{R_{1}^{2} R_{2}^{2}} \mathrm{~d} A
$$

where $P_{r}$ is the received clutter power, $P_{t}$ is the transmitted power, $G_{t}$ and $G_{r}$ are the transmit and receive antenna gains, $\lambda$ is the wavelength, and $R_{1}$ and $R_{2}$ are the ranges from the transmitter and receiver to the target [7]. If there is a relatively large clutter source such as an antenna support structure in the clutter ellipse containing the desired target, Equation (1) says it will be integrated into the clutter power competing with the desired target return.

Common clutter suppression techniques such as range gating and coherent background subtraction may be ineffective if the target has strong bistatic scattering lobes which interact with these background structures. Since the exact interaction geometry is target dependent and, in the case of a variable bistatic angle measurement, angle dependent, it is difficult to design an absorber treatment that is equally effective for all measurement geometries. The only alternative is to create a non-interacting support mechanism for the bistatic receiving channel. The next section presents just such a minimally interacting support mechanism and describes an experiment to demonstrate its advantage over traditional bistatic channel designs.

\section{CONCEPT DEMONSTRATION}

The basic concept proposed is simply to replace the ridged antenna support structure commonly used in bistatic measurement ranges with a low scatter string support system. The scattering characteristics of high tensile strength dielectric strings have been shown sufficient for low RCS measurements while being able to support significant target loads [4]. Until recently however, the inherent instability of a suspension type support system limited their use as compared to ogival pylons and foam columns. Buterbaugh etal. [3] have overcome this limitation in their inverted Stewart platform design which we adapt here.

One of the challenges in moving from target support to supporting an active bistatic antenna receiver is the need to get the received signal back to the radar without re-introducing scattering structures such as RF cables into the range. The author's approach is to use a wide-band RF-to-optical modem to convert the received RF signal to an optical signal which can be sent to the receiver via a relatively small, low scattering fiber optic cable. Thus the only significant scattering structure in the range is the actual receive antenna and a small box housing the LNA, modem, and battery power supply (see Figure 2).

In order to demonstrate the viability of this new method, the author directed a comparative experiment whereby the same target was measured in AFIT's RCS measurement range using the traditional bistatic arm and the proposed string antenna support system. The experiment was designed specifically to keep all variables the same except the clutter introduced

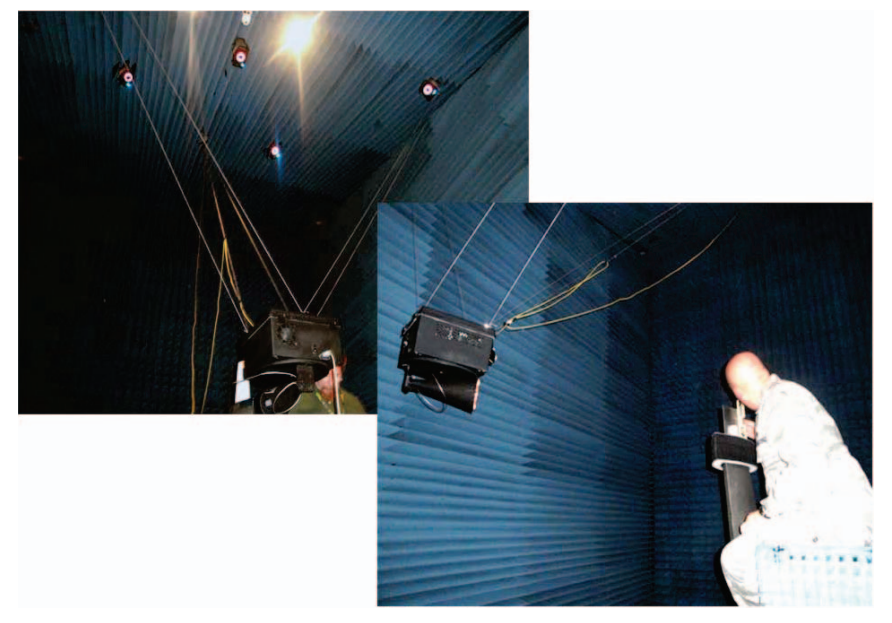

Fig. 2. Battery powered bistatic receive channel with a quad-ridge, wide band antenna. Also shown is the ceiling mounted optical position measurement system used to control the position and orientation of the receive antenna.

by the bistatic receiver support system. A description of the experiment and qualitative analysis of representative results follows.

\section{A. AFIT's RCS Measurement Facility}

The AFIT RCS measurement range is an anechoic chamber with a tapered design approximately 14'x16' at the throat, 24 'x26' at the back wall, and $45^{\prime}$ in length. The facility is designed primarily for academic research and thus is fairly flexible, being able to be configured as a far-field or compact range with either ogive pylon, foam column, or string target support systems. When configured for bistatic measurements, the bistatic arm/string support system can be used in conjunction with the latter target support mechanisms to provide either swept or fixed angle bistatic measurements. The range uses a Lintek 4000, PNA based pulsed CW radar with a frequency coverage from 2-18 GHz. For the subject demonstration, Flamm \& Russell FR6400 low side lobe diagonal horns were used in the transmit channel to minimize clutter illumination. The penalty was the reduction in frequency range to $6-18 \mathrm{GHz}$ due to antenna cutoff.

\section{B. Experiment Design}

As the main purpose of the demonstration is to compare the impact of bistatic clutter on an RCS measurement for the two support systems, the author chose a target with a fairly wide dynamic range and relatively large bistatic signature. The 6" square metal plate served the purpose for the size and frequency range available in AFIT's range. The author used horizontal $(\phi \phi)$ polarization for the resulting low RCS near the edge-on illumination angles. For the sake of brevity, only a single fixed angle bistatic measurement is reported here. A more extensive examination of both fixed and variable angle bistatic measurements of a variety of interacting targets is presented in a companion journal paper currently in the submission process. 
For both the traditional bistatic arm measurement and the string support measurement, the standard RCS calibration procedure as expressed in Equation (2) is performed. Specifically the calibrated bistatic RCS is defined by,

$$
\sigma=\frac{\sigma_{t a r}-\sigma_{b k g}}{\sigma_{c a l}-\sigma_{c b k}} \cdot \sigma_{t h r}
$$

where the tar subscript denotes the target measurement, bkg denotes target background, cal denotes calibration object, $c b k$ denotes calibration background, and thr denotes the theoretical calibration object bistatic RCS. The calibration was verified using the procedure recommended in [8], resulting in measurement uncertainties of $\pm 0.75 \mathrm{dBsm}$.

In the two measurements presented, the bistatic receiver was positioned $20^{\circ}$ from the transmitter approximately 8 ' from the target. The target was mounted on a low RCS ogival pylon and rotated $360^{\circ}$ about an axis in the plane of the plate and perpendicular to the plane formed by the transmit and receive legs (see Figure 3).

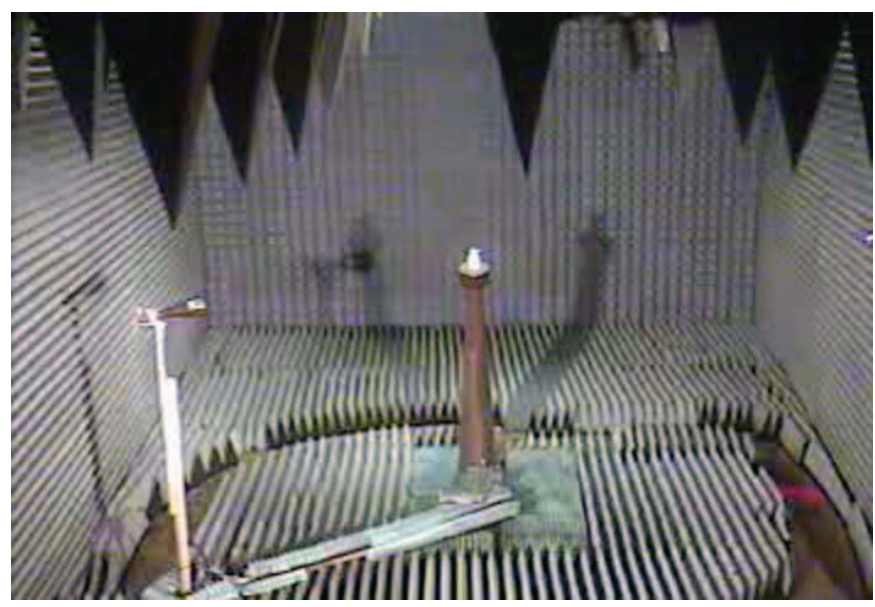

Fig. 3. AFIT's RCS measurement range configured with a quad-ridge, wide band horn mounted on the bistatic arm. The 6" square plate (not shown) is mounted on the short foam column atop the low RCS ogival pylon. The transmit antenna orientation is the same as the camera except it is in the same plane as the bistatic antenna and target height.

\section{Comparative Data Analysis}

We begin the analysis by examining the global RCS plots shown in Figures 4 and 5. As presented, the global RCS represents the frequency dependent bistatic scattering as a function of the plate's angle with respect to the illumination direction. The inner radius corresponds to a frequency of $6 \mathrm{GHz}$ and the outer $18 \mathrm{GHz}$. From both figures we see a peak RCS response at $170^{\circ}$ and $350^{\circ}$. This is consistent with the $20^{\circ}$ fixed bistatic angle and relative position of the receive antenna as the plate rotated. We also note the peak values are of equal magnitude in $\mathrm{dBsm}$, indicating a relatively high signal to clutter level.

Turning our attention to the lower RCS sectors of the plots, we see a distinct difference in scattering behavior. While the

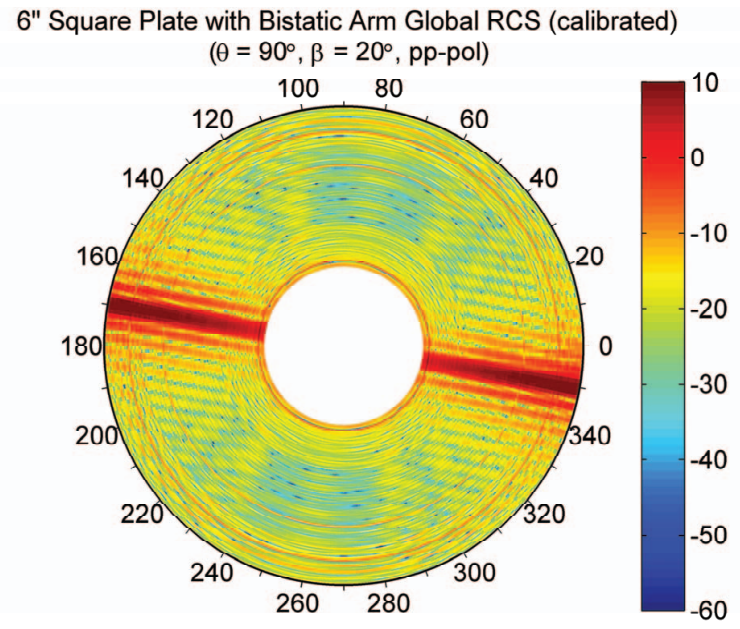

Fig. 4. Global RCS for 6" square plate with a fixed bistatic angle $(\beta)$ of $20^{\circ}$ where the receiver is supported by the ridged support tower.

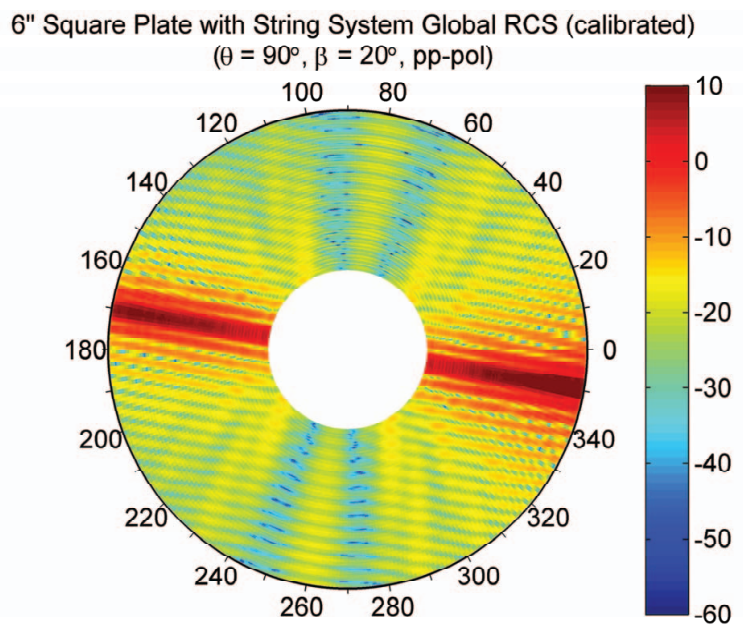

Fig. 5. Global RCS for 6" square plate with a fixed bistatic angle $(\beta)$ of $20^{\circ}$ where the receiver is supported by low scatter dielectric strings.

plate measured with the string support system shows the distinctive lobing structure associated with near grazing incidence angles, the traditional bistatic arm exhibits less lobing and a higher overall RCS level indicative of an interfering source. We also note a few discrete frequencies with what appears to be radio frequency interference (RFI) in the bistatic arm measurement of Figure 4. Clearly, there is a higher clutter level in the bistatic arm measurements. To better recognize the source of this clutter, we next present the associated global 


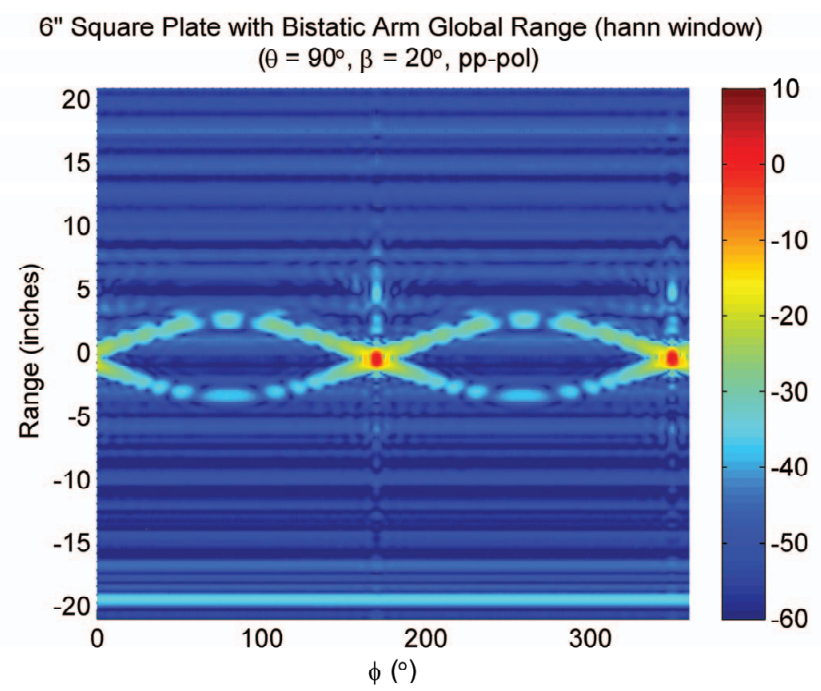

Fig. 6. Global Range for 6" square plate with a fixed bistatic angle $(\beta)$ of $20^{\circ}$ where the receiver is supported by the the ridged support tower.

range plots generated by concatenating a series of down range images at each measurement angle.

Figures 6 and 7 tell a consistent story in that the bistatic arm measurements contain a significantly higher clutter level with a notable peak at approximately 20" up range from the plate. For the most part the clutter seems to be independent of plate angle indicating a direct clutter source rather then a target/background interaction term. Both plots show the distinctive pattern produced by the edge scattering from the plate as it rotates. The weaker scattering from the leading edge (relative to the illumination direction) is consistent with horizontal polarization scattering. Both plots also show the nulls in the trailing edge scatter as the plate comes into alignment with either the transmit antenna or the receive antenna separated by $20^{\circ}$. However, the string support system measurement has none of the nulling in the leading edge return caused by the bistatic clutter in the bistatic arm measurement. This is why the lobing structure around plate edge-on illumination angles is missing in the corresponding global RCS plot (Figure 4). The two point interference interference between the leading and trailing edges is destroyed.

Clearly, the proposed string antenna support system is a significantly less interfering alternative to the traditional mechanical towers. The inverted Stewart platform design also gives equal mechanical stability with six degrees of freedom for antenna orientation. Perhaps the most significant disadvantage to the proposed methodology is limited battery life. Our current prototype system requires a recharge every 1.5 hours.

\section{CONCLUSION}

A novel low clutter antenna support method for bistatic RCS measurements is proposed with significant benefits over

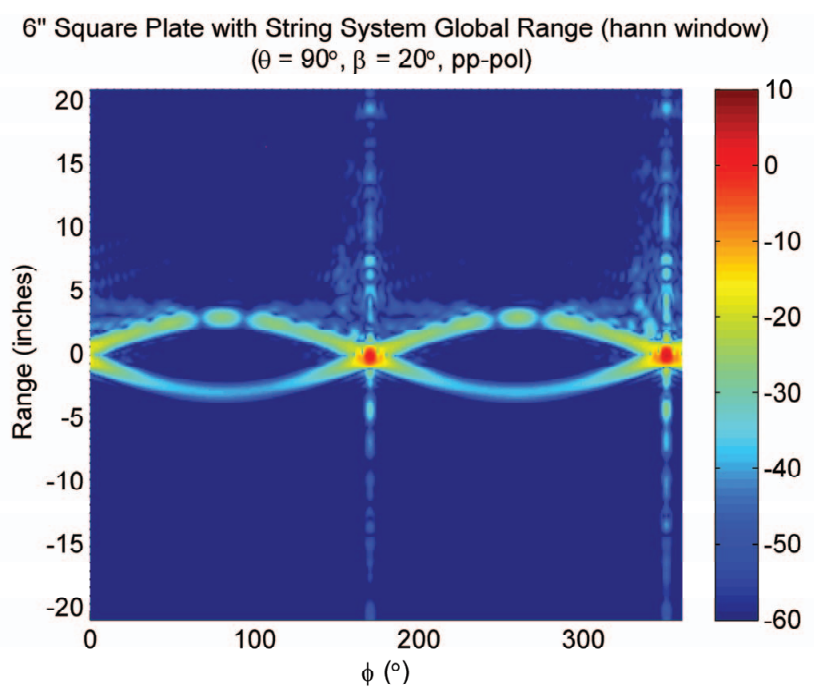

Fig. 7. Global Range for 6" square plate with a fixed bistatic angle $(\beta)$ of $20^{\circ}$ where the receiver is supported by low scatter dielectric strings.

traditional mechanical means. We present the results of a small experiment designed to compare the influence of antenna support clutter on a bistatic RCS measurement. While not an exhaustive quantitative examination, the qualitative results clearly demonstrate the superiority of the proposed method. A more extensive quantitative examination is forthcoming in a future journal publication.

\section{ACKNOWLEDGMENT}

The author would like to thank Dr. Brian Kent of the Air Force Research Laboratory for discussions which planted the seed of the idea and my two masters students, William Gunn and Jonathan Spaulding, who helped water it by performing some of the measurements reported here.

\section{REFERENCES}

[1] N. J. Willis, Bistatic Radar, 2nd ed. Maryland, USA: Technology Service Corporation, 1995

[2] H. D. Griffiths, "From a different perspective: principles, practice and potential of bistatic radar," Proceedings of the International Radar Conference, pp.1-7, 2003.

[3] A. Buterbaugh and C Mentzer, "Critical Technologies for Performing RCS Target Measurements using a String Support System," Proceedings of the Antenna Measurement Techniques Association, November, 2002.

[4] A. Buterbaugh, B. M. Kent, C. Mentzer, M. Scott, and W. Forster, "Demonstration of an Inverted Stewart Platform Target-Suspension System Using Lightweight, High-Tensile Strings," IEEE Antennas and Propagation Magazine, Vol. 49, No. 5, October 2007.

[5] IEEE Standard Radar Definitions, IEEE Std. 686-2008, 21 May 2008.

[6] IEEE Recommended Practice for Radar Cross-Section Test Procedures, IEEE Std. 1502-2007, 7 September 2008.

[7] N. C. Currie, Radar Reflectivity Measurement: Techniques and Applications, 1st ed. Norwood, MA: Artech House, 1989.

[8] B. M. Kent, 'Comparative measurements of precision radar cross section (RCS) calibration targets," IEEE International Symposium of the Antennas and Propagation Society, Vol. 4, pp. 412-415, 2001. 